\title{
Destroy to Save
}

\author{
Geoffroy de Clippel $^{1}$ \\ Department of Economics, Brown University, Providence, RI, USA \\ Victor Naroditskiy ${ }^{2, *}$ \\ School of Electronics and Computer Science, University of Southampton, UK \\ Maria Polukarov ${ }^{2, *}$ \\ School of Electronics and Computer Science, University of Southampton, UK \\ Amy Greenwald* \\ Department of Computer Science, Brown University, Providence, RI, USA \\ Nicholas R. Jennings ${ }^{2, *}$ \\ School of Electronics and Computer Science, University of Southampton, UK \\ Department of Computing and Information Technology, King Abdulaziz University, \\ Saudi Arabia
}

\begin{abstract}
We study the problem of allocating $m$ identical items among $n>m$ agents with unit demand and private value for consuming the good. We allow payments and focus on dominant-strategy implementation. In the absence of an auctioneer who can absorb payments collected from the agents, the payments must be burnt to support dominant-strategy implementation.

Recent work modified the classic VCG mechanism by redistributing as

\footnotetext{
${ }^{*}$ Corresponding author.

Email addresses: declippel@brown.edu (Geoffroy de Clippel),

${ }^{1}$ Financial support from the National Science Foundation (grant SES-0851210) and the

${ }^{2}$ The authors gratefully acknowledge funding from the UK Research Council for project
} vn@ecs.soton.ac.uk (Victor Naroditskiy), mp3@ecs.soton.ac.uk (Maria Polukarov), amy@brown.edu (Amy Greenwald), nrj@ecs.soton.ac.uk (Nicholas R. Jennings) Deutsche Bank through the Institute for Advanced Study is gratefully acknowledged. ORCHID, grant EP/I011587/1.
\end{abstract}


much of the payments as possible back to the agents while still satisfying incentive constraints. This approach guarantees allocative efficiency, but in some cases a large percentage of social welfare is lost. In this paper, we provide a mechanism that is not allocatively efficient but is instead guaranteed to achieve at least $80 \%$ of the social welfare as $n \rightarrow \infty$. Moreover, in the extreme case of $m=n-1$ where VCG-based mechanisms provide zero welfare, the percentage of social welfare maintained by our mechanism asymptotically approaches $100 \%$.

\section{Introduction}

Suppose six city-dwelling roommates jointly own a car that seats five people. They decide to take a trip to the countryside. While they all would like to go, there is not room for all of them in the car. Some need the fresh country air while others would not really mind staying home. The roommates do not necessarily know one another's desires, but each of them knows her own true value of getting out of the city. How should they decide who gets to go?

Against this background, we study a class of resource allocation problems, in which $m$ identical items need to be distributed among $n>m$ agents. Each agent wants exactly one item, and has a private value for that item. We assume the agents can make monetary payments, and have quasi-linear utilities. Under these assumptions, agents can be incentivized to reveal their private values truthfully. Unlike traditional auction settings, we focus on scenarios with no auctioneer. Such scenarios include the allocation of university parking spots among faculty members, free tickets for a sporting event among club members, or seats on an overbooked airplane. In the presence of a participant possessing no private information (e.g., an auctioneer), monetary payments can be absorbed by him, thus achieving budget balance. In our setting however, all agents have private information, and any collected payments need to be burnt in order to maintain truthful reporting. Burning money is undesirable as it decreases social welfare. Therefore, it is important to design mechanisms that ensure a high level of social welfare while maintaining the incentives.

In this work, we focus on strategy-proof mechanisms, which require that it be a dominant strategy for each agent to report her value truthfully. This requirement is less permissive, but more robust than Bayesian implementation. In particular, agents are more likely to play a dominant strategy than a 
strategy that is optimal only when other agents play their part of the truthful equilibrium. Perhaps even more importantly, dominant-strategy implementation does not require any assumptions about the distribution of the agents' values (or their beliefs about those values), nor their attitude towards risk.

As for measuring the appeal of various strategy-proof mechanisms, we choose the strictest and most robust metric: worst-case performance. More specifically, if one fixes the agents' profile of values, one can compute the ratio of the social welfare realized by the mechanism over the maximal social welfare that could be achieved, should these values be known. Since these values are not known, nor is their probabilistic distribution, the appeal of a strategy-proof mechanism is measured by the minimum of this ratio over all possible value profiles. We call this ratio a social welfare ratio, ${ }^{3}$ and we use it to determine a mechanism's worst-case (i.e., guaranteed) level of social welfare: reaching a level $\alpha \in[0,1]$ means that a mechanism realizes at least a proportion $\alpha$ of the maximal social welfare for every possible profile of the agents' values.

In addition to strategy-proofness, we also impose the following natural constraints: 1) feasibility - no more than $m$ items can be allocated, and monetary deficits are not allowed (i.e., no external subsidy), 2) individual rationality - each agent's total utility is nonnegative, and 3) anonymity - the allocation and payment decision applied to each agent does not depend on her identity. The question we are interested in can now be stated formally:

Find a mechanism that maximizes the worst-case social welfare ratio among all those that are strategy-proof, feasible, individually rational, and anonymous.

Recently, two sets of authors (Moulin (2009) and Guo and Conitzer (2009)) solved the above question under the additional constraint that the items be allocated to the $m$ agents who value them most. Their solution characterized the best mechanism within the class of Groves mechanisms, ${ }^{4}$ which has received special attention in the economics literature because members of this class admit a simple functional form (cf. characterization by Green and Laffont (1979)). A Groves mechanism guarantees an efficient allocation of all

\footnotetext{
${ }^{3}$ The same measure is used in (Moulin, 2009; Guo and Conitzer, 2009).

${ }^{4}$ The most well-known Groves mechanism is the VCG mechanism. Here, the terms Groves mechanisms and VCG redistribution mechanisms are used interchangeably.
} 
$m$ items, but not necessarily a good level of "overall" efficiency (as measured for instance by the worst-case social welfare ratio), because allocative efficiency may come at the cost of "burning" quite a bit of money to meet the incentive constraints, whereas it may be better in terms of overall efficiency to destroy some items in order to burn less money. Indeed, one can easily check that it is impossible to guarantee a strictly positive worst-case social welfare ratio using a Groves mechanism when $m=n-1$, while applying the best Groves mechanism after destroying one item would secure a strictly positive ratio.

Still, applying a Groves mechanism after destroying some fixed number of items is not the best way to optimize overall efficiency. Rather, we show that contingent destruction mechanisms, which make destruction decisions based on the values agents report, perform better. In fact, all strategy-proof mechanisms rely on contingent payments, as well as contingent destruction: an agent receives an item if and only if her reported value is larger than a threshold value, which in general depends on other agents' reports. We refer to such mechanisms as threshold mechanisms.

The question of finding the best threshold mechanism is more complicated than the question of finding the best Groves mechanism. The Groves mechanism's allocation function is constant once the agents' values are ordered (the agents with the $m$ highest values receive the items). In this restricted context, Guo and Conitzer (2009) and Moulin (2009) calculate the optimal payment function. In contrast, allocation functions in the broader class of threshold mechanisms are not constant.

Our approach is designed to achieve the right balance between tractability, and showing that one can obtain a significant improvement in overall welfare if one does not rely on the technical convenience of Groves mechanisms. Even a very minor departure from VCG - destroying at most one item-allows for drastic savings in problem instances with many agents and items. Importantly, the ratio guarantee improves as the number of items increases. Perhaps most striking is the case where $m=n-1$. As already pointed out, Groves mechanisms do not provide any strictly positive ratio in this case. Further, applying the best Groves mechanism after destroying a fixed number of items does not guarantee a ratio larger than $1 / 2$ (see numerical computations in Guo and Conitzer (2008)). In contrast, our method of contingent destruction guarantees the ratio of $1-\frac{2}{n^{2}-n}$, which rapidly approaches 1 as $n$ increases. Finally, an additional advantage of our mechanism is that it has a much simpler analytical form than the optimal Groves 
mechanism.

In more detail, our threshold mechanism, termed SimpleDestroy, charges each allocated agent her threshold. If the total payment is low, all items are allocated efficiently. If the total payment is high, then one item is destroyed, the remaining items are allocated efficiently, and most of the payments are redistributed back to the agents. SimpleDestroy complements the optimal efficient mechanism (Moulin, 2009; Guo and Conitzer, 2009): the former performs best when the number of items is high, while the ratio of the latter decreases with the number of items. At the respective extremes each mechanism performs poorly: the ratio of SimpleDestroy is zero for $m=1$, and the ratio of the optimal efficient mechanism is zero for $m=n-1$. However, using the better of the two mechanisms can provide good performance guarantees for all $m$ and $n$. In fact, we provide a hybrid mechanism that guarantees the ratio of .8 as $n$ increases and $m=\alpha n$ for any $\alpha \in(0,1)$.

We discuss related literature next. Optimal efficient mechanisms have recently been studied in a series of papers. Mechanisms that are applicable in any setting where VCG can be used have been proposed by Bailey (1997) and Cavallo (2006). Only the latter mechanism is guaranteed to be budgetbalanced in all settings. As already mentioned, Guo and Conitzer (2009) and Moulin (2009) independently discover the optimal VCG redistribution mechanism for the allocation domain studied here. Guo and Conitzer (2010b) derive a linear redistribution Groves mechanism to maximize the expected social welfare when the distribution of agents' values is known. Porter et al. (2004) study the problem of allocating undesirable goods (e.g., tasks) to agents in a fair manner. In the model where a single item (task) needs to be allocated, the mechanisms by Porter, Bailey, and Cavallo coincide. ${ }^{5}$ Naroditskiy et al. (2012) provide a characterization of optimal redistribution functions in single-parameter domains, and extend the mechanism by Porter et al. (2004) to multiple tasks. Optimal redistribution when heterogeneous items are allocated have been conjectured by Gujar and Narahari (2011) and proved by Guo (2012).

Welfare maximization has also been considered in randomized mechanisms. Faltings (2005), for instance, proposes a generally-applicable mechanism that picks an agent at random, excludes him from allocation, and makes

\footnotetext{
${ }^{5} \mathrm{~A}$ detailed discussion of relationship between these mechanisms appears in (Guo and Conitzer, 2009).
} 
him the recipient of the VCG payments. The mechanism (also studied in Guo and Conitzer (2008); Guo et al. (2011)) achieves budget balance, but loses efficiency for value profiles where the excluded agent's value is high enough to be allocated. The guaranteed ratio is $\frac{n-1}{n}$, however this is a somewhat weaker metric as the ratio now is expected over the random choice of the excluded agent.

Most related to our work is the work on randomized mechanisms by Guo and Conitzer (2008). Starting from the observation that destroying items might save enough money to be socially beneficial, they limit attention to allocation rules where the number of items destroyed is independent of the agents' reports. In order for the social benefit to be more significant, they allow for that number to be determined randomly. Instead, we observe that significant gains can be achieved via deterministic mechanisms provided one uses contingent destruction rules. Furthermore, Guo and Conitzer require feasibility only in expectation, and need to assume that the agents are risk neutral. Our deterministic analysis dispenses with these assumptions. If one is willing to use lotteries, then it may be of interest to combine the insights from our two papers, making Guo and Conitzer's random variables depend on reported values.

A preliminary version of this paper appeared in ACM EC 09 (de Clippel et al., 2009). The results in Section 4, which are the main analytical contribution of this paper, are novel.

This paper unfolds as follows. Section 2 formally states the problem we are studying. A computational method of searching for an optimal mechanism in a restricted setting is proposed and applied in Section 3. Based on the numerical results, we discern the analytical form of a general mechanism in Section 4.

\section{Definitions}

An allocation problem is a triple $\langle n, m, v\rangle$, where $n$ is the number of agents, $m<n$ is the number of (identical) items available to allocate, and $v \in \mathbb{R}_{+}^{n}$ represents the agents' satisfaction from consuming one item (agents do not care for consuming multiple units). We restrict attention to value profiles $v$ such that $v_{1} \geq v_{2} \geq \ldots \geq v_{n} \geq 0$. This is without loss of generality since our problem involves only anonymous mechanisms. Monetary compensations are possible, and utilities are quasi-linear. The space of possible value profiles is then $V=\left\{v \in \mathbb{R}_{+}^{n} \mid v_{1} \geq v_{2} \geq \ldots \geq v_{n} \geq 0\right\}$. An allocation is a pair 
$(\mathbf{f}, \mathbf{t}) \in\{0,1\}^{n} \times \mathbb{R}^{n}$, where $\mathbf{f}_{i}=1$ if and only if agent $i$ gets one item, and $\mathbf{t}_{i}$ represents the amount of money that agent $i$ receives (this number can be negative, of course, in which case agent $i$ pays that amount). Hence, the total utility of agent $i$ when implementing the allocation $(\mathbf{f}, \mathbf{t})$ is $\mathbf{f}_{i} v_{i}+\mathbf{t}_{i}$, if her value for an item is $v_{i}$. A mechanism is a pair of functions $f: \mathbb{R}_{+}^{n} \rightarrow\{0,1\}^{n}$ and $t: \mathbb{R}_{+}^{n} \rightarrow \mathbb{R}^{n}$. Thus, it determines an allocation for each possible report from the agents regarding their value for an item. We slightly abuse the notation and define $f_{i}(v)=(f(v))_{i}$ and $t_{i}(v)=(t(v))_{i}$. The vector $v_{-i} \in \mathbb{R}^{n-1}$ denotes values of the agents other than agent $i$ and the vector $v$ can be written as $\left(v_{i}, v_{-i}\right)$. We focus on mechanisms that satisfy the following constraints:

- Feasibility: no more than $m$ items should be allocated, and the sum of payments to the agents should be less than or equal to zero, for all value profiles $v$.

$$
\sum_{i=1}^{n} f_{i}(v) \leq m \text { and } \sum_{i=1}^{n} t_{i}(v) \leq 0 \quad \forall v \in V
$$

- Strategy-proofness: it is a dominant strategy for each agent to report her value truthfully.

$$
f_{i}\left(v_{i}, v_{-i}\right) v_{i}+t_{i}\left(v_{i}, v_{-i}\right) \geq f_{i}\left(v_{i}^{\prime}, v_{-i}\right) v_{i}+t_{i}\left(v_{i}^{\prime}, v_{-i}\right) \quad \forall v \in V, i, v_{i}^{\prime}
$$

- Individual Rationality: it is in each agent's interest to participate in the mechanism, for all value profiles $v$.

$$
f_{i}(v) v_{i}+t_{i}(v) \geq 0 \quad \forall v \in V, i
$$

We now define the index that we use to measure the overall efficiency of a mechanism $(f, t)$ that is implemented truthfully (an equivalent index was used in (Moulin, 2009; Guo and Conitzer, 2009, 2008)). If the true value profile is $v$, then the social welfare realized by the mechanism is equal to $\sum_{i=1}^{n}\left[v_{i} f_{i}(v)+t_{i}(v)\right]$. However, this number is not informative on its own. Instead, we would like to know how far it is from the first-best solution, i.e. from the maximal welfare one could achieve if the agents' values were known. In order to have an index that is unit-free (i.e., homogenous of degree zero), it is natural to consider a ratio. Finally, since the agents' values are not known, nor their probabilistic distribution, it is natural to consider the worst-case 
index. To summarize, the index that we use to measure the performance of a mechanism $(f, t)$ that is truthfully implemented is given by the following ratio:

$$
\min _{v \in V} \frac{\sum_{i=1}^{n}\left[f_{i}(v) v_{i}+t_{i}(v)\right]}{\max _{f^{\prime} \in \mathcal{F}(m)} \sum_{i=1}^{n} f_{i}^{\prime} v_{i}},
$$

where $\mathcal{F}(m)=\left\{f^{\prime} \in\{0,1\}^{n} \mid \sum_{i=1}^{n} f_{i}^{\prime} \leq m\right\}$. Finding a mechanism whose ratio is $\beta$ means that a proportion $\beta$ of the maximal social welfare is achieved, independently of what the true values are. Following the convention $v_{1} \geq$ $v_{2} \geq \ldots \geq v_{n} \geq 0$, the denominator becomes $\sum_{i=1}^{m} v_{i}$ and we write the ratio $\mathrm{as}^{6}$

$$
\min _{v \in V} \frac{\sum_{i=1}^{n}\left[f_{i}(v) v_{i}+t_{i}(v)\right]}{\sum_{i=1}^{m} v_{i}}
$$

The formal content of the question stated in the introduction can thus be summarized by the following optimization problem:

$$
\begin{aligned}
& \max _{(f, t)} \quad \min _{v \in V} \frac{\sum_{i=1}^{n}\left[f_{i}(v) v_{i}+t_{i}(v)\right]}{\sum_{i=1}^{m} v_{i}} \\
& \sum_{i=1}^{n} f_{i}(v) \leq m \quad \forall v \in V \\
& \sum_{i=1}^{n} t_{i}(v) \leq 0 \quad \forall v \in V \\
& f_{i}\left(v_{i}, v_{-i}\right) v_{i}+t_{i}\left(v_{i}, v_{-i}\right) \geq f_{i}\left(v_{i}^{\prime}, v_{-i}\right) v_{i}+t_{i}\left(v_{i}^{\prime}, v_{-i}\right) \quad \forall v \in V, i, v_{i}^{\prime} \\
& f_{i}(v) v_{i}+t_{i}(v) \geq 0 \quad \forall v \in V, i
\end{aligned}
$$

The problem above can be simplified using the characterization of dominantstrategy implementable mechanisms in settings where agent's private information is a single number $v_{i} \in \mathbb{R}$.

Theorem 1 (e.g., see Nisan et al. (2007) p. 229). A mechanism $(f, t)$ is implementable in dominant strategies if and only if for each agent $i$ : (i) $f_{i}$

\footnotetext{
${ }^{6}$ The allocation problem is trivial if $\sum_{i=1}^{m} v_{i}=0$. Without loss of generality, we assume $\sum_{i=1}^{m} v_{i}>0$.
} 
is monotone in $_{i}$; (ii) $t_{i}(v)=h\left(v_{-i}\right)-\tau\left(v_{-i}\right)$ if $f_{i}(v)=1$ (i.e., $i$ is allocated) and $t_{i}(v)=h\left(v_{-i}\right)$ otherwise, where $\tau\left(v_{-i}\right)=\sup _{v_{i} \mid f_{i}\left(v_{i}, v_{-i}\right)=0} v_{i}$ defines the threshold. ${ }^{8,9}$

These mechanisms are easy to interpret. Each agent faces a personalized price (the threshold $\tau$ ) that is determined by the reports of the other agents. She gets the good if and only if her reported value is larger than this price, and must pay it in exchange. The collected money can be redistributed to some extent to the agents via the rebate function $h$. The Groves mechanisms form a special case, where $i$ 's price is the $m^{\text {th }}$ largest component of $v_{-i}$.

The allocation function $f$ is determined by the threshold function, while the payment function $t$ is determined by the threshold and rebate functions. Thus, we can restate the optimization problem (1)-(5) using these functions.

$\max _{(h, \tau)} \min _{v \in V} \frac{\sum_{i \mid v_{i} \geq \tau\left(v_{-i}\right)}\left(v_{i}-\tau\left(v_{-i}\right)\right)+\sum_{i=1}^{n} h\left(v_{-i}\right)}{\sum_{i=1}^{m} v_{i}}$
$\#\left\{i \mid v_{i} \geq \tau\left(v_{-i}\right)\right\} \leq m \quad \forall v \in V$
$\sum_{i=1}^{n} h\left(v_{-i}\right) \leq \sum_{i \mid v_{i} \geq \tau\left(v_{-i}\right)} \tau\left(v_{-i}\right) \quad \forall v \in V$
$h\left(v_{-i}\right) \geq 0 \quad \forall v \in V, i$

The first constraint is the feasibility constraint with respect to the items being allocated ${ }^{10}$, while the second constraint is the feasibility constraint

\footnotetext{
${ }^{7}$ Monotonicity of $f_{i}$ in $v_{i}$ means that if an agent is allocated when she reports $v_{i}$, she is also allocated when she reports $v_{i}^{\prime} \geq v_{i}$.

${ }^{8} \mathrm{An}$ agent $i$ is allocated if and only if her report is above the threshold $\tau\left(v_{-i}\right)$.

${ }^{9}$ As our focus is on anonymous mechanisms, the theorem was adapted to payment functions $h$ (rather than $h_{i}$ ) that do not vary from agent to agent.

${ }^{10}$ For notational convenience, from now on we restrict our attention to profiles $v$ where all components are distinct. This restriction is introduced without loss of generality as we can extend the mechanism to all value profiles by using uniform lotteries to break ties, as is usually done in papers on auctions. Suppose for instance that agent $i$ should receive an item, and that more than $m$ other agents have the same value as $i$. Anonymity would then conflict with feasibility. A uniform lottery can then be used to determine which subset of agents receives an item, among all those that have the same value. Even so, the way agents react to risk is irrelevant because all the outcomes of the lottery are equivalent in
} 
with respect to money (the sum of all rebates should be no more than the amount collected from the agents that get an item). The third constraint is the individual rationality constraint (remember that an agent's value must be larger than the threshold when she gets an item, and so this constraint is trivially satisfied for her as well). The strategy-proofness constraint is no longer needed as all threshold mechanisms are strategy-proof.

We now propose a last formulation of our optimization problem. We remove the minimization over $v$ by introducing a variable $r \in \mathbb{R}$ denoting the best ratio that holds for any profile of values. The resulting optimization program appears in Figure 1.

$$
\begin{aligned}
& \max _{(h, \tau), r} r \\
& \sum_{i \mid v_{i} \geq \tau\left(v_{-i}\right)}\left(v_{i}-\tau\left(v_{-i}\right)\right)+\sum_{i=1}^{n} h\left(v_{-i}\right) \geq r \sum_{i=1}^{m} v_{i} \quad \forall v \in V \\
& \#\left\{i \mid v_{i} \geq \tau\left(v_{-i}\right)\right\} \leq m \quad \forall v \in V \\
& \sum_{i=1}^{n} h\left(v_{-i}\right) \leq \sum_{i \mid v_{i} \geq \tau\left(v_{-i}\right)} \tau\left(v_{-i}\right) \quad \forall v \in V \\
& h\left(v_{-i}\right) \geq 0 \quad \forall v \in V, i
\end{aligned}
$$

Figure 1: The problem of finding an optimal mechanism stated as an optimization problem.

A solution to the mathematical program above is the optimal mechanism for the allocation domain. ${ }^{11}$ However, the program is hard to solve both analytically and computationally for the following reasons. Firstly, maximization is over arbitrary functions $h$ and $\tau$. Secondly, the program has an infinite number of constraints as the set of possible value profiles $v \in V$ is infinite. Thirdly, the constraints include indicator variables: for example, the summation on the right-hand side of Equation 9 can equivalently be stated as $\sum_{i} \tau\left(v_{-i}\right) \mathbf{1}_{\left\{v_{i} \geq \tau\left(v_{-i}\right)\right\}}$. In the next section we tackle the problem compu-

terms of utility. Specifically, the lottery is between receiving an item worth $v_{i}$ at a price $p_{i}$ and receiving a rebate $h_{i}$ such that $h_{i}=v_{i}-p_{i}$.

${ }^{11} \mathrm{~A}$ lower bound on the objective value is given by the ratio of the optimal efficient mechanism (Moulin, 2009; Guo and Conitzer, 2009). 
tationally. Guided by the computational results, in Section 4 we propose a closed-form mechanism and prove that it has high performance guarantees.

\section{Numerical Approach and Results}

This section uses a computational approach to find heuristic solutions to the problem in Figure 1. We make assumptions restricting the space of solutions to make the problem computationally tractable, and find optimal mechanisms within the restricted class. Although not generally optimal, these solutions have high performance guarantees providing significant improvement over known mechanisms. The steps we take to restrict the solution space and the numerical results help identify a simple and well-performing mechanism, which we present in Section 4.

There are infinitely many constraints in the optimization problem in Figure 1 and the problem is non-linear due to indicator variables. However, notice that if we fix a threshold function $\tau$ and only optimize over rebate functions, the indicator variables disappear, and the optimization problem becomes a linear program. This linear program cannot be solved directly as it has an infinite number of constraints - one for each possible value profile $v \in V$. To deal with this, we observe that if one can subdivide the value space into (convex) regions where the constraints are linear, then it is enough to enforce constraints only at the extreme points of each region. ${ }^{12}$ We restrict attention to the threshold and rebate functions for which we can partition the space of value profiles into such regions.

Letting $w \in \mathbb{R}^{n-1}$ refer to a profile of agents' values with one agent excluded, we make two assumptions.

Assumption 1. The threshold function is of the form $\tau(w)=\max \left(k w_{p}, w_{m}\right)$, with $k \in[0,1]$ and $p \in\{1,2, \ldots,(m-1)\}$.

Adding the $w_{m}$-component inside the max operator guarantees that no more than $m$ items are allocated, as required by the feasibility constraint. The $p$ parameter determines how many items are guaranteed to be allocated, independently of the agents' reports. The parameter $k$ controls how large the values of agents $p+1$ through $m$ must be for them to be allocated.

\footnotetext{
${ }^{12}$ The ideas underlying this technique have been proposed in (Guo and Conitzer, 2008) and generalized in (Naroditskiy et al., 2012; Dufton et al., 2012).
} 
Taking $k=0$ brings us back to Groves mechanisms, while setting of $k=1$ means that items $p+1, \ldots, m$ are always destroyed.

For a given pair of parameters $(k, p)$, the threshold function $\tau(w)=$ $\max \left(k w_{p}, w_{m}\right)$ is linear on two regions:

$$
\tau(w)= \begin{cases}k w_{p} & \text { if } k w_{p} \geq w_{m} \\ w_{m} & \text { otherwise }\end{cases}
$$

We restrict the rebate function to be linear on the same regions.

Assumption 2. The rebate function $h$ is linear in values on two regions:

$$
h(w ; a, b)= \begin{cases}a w & \text { if } k w_{p} \geq w_{m} \\ b w & \text { otherwise }\end{cases}
$$

where $a, b \in \mathbb{R}^{n-1}$.

The approach we follow to optimize over the class of mechanisms satisfying these assumptions is summarized in Figure 2. We consider threshold functions satisfying Assumption 1 with $k$ coming from a finite set of constants between 0 and 1 and $p$ taking any value between 1 and $m-1$. For each threshold function specified by $k$ and $p$, we compute the rebate function that guarantees the highest social welfare among rebate functions satisfying Assumption 2. We then select the threshold and the corresponding rebate functions that achieved the highest welfare among the ones we considered. The key step is computation of optimal rebates, which is the focus of the rest of this section.

To tackle computation of rebates, we first characterize the regions where the rebate function is linear and the number of allocated items is constant. By the definition of the threshold function $\tau=\max \left(k w_{p}, w_{m}\right)$, there are $m-p+1$ possible allocations (the first $p$ agents get the items, the first $p+1$ agents get the items, ..., the first $m$ agents get the items) determined by the position of $k v_{p}$ among $v_{p} \ldots v_{m}$. The rebate function $h(w)$ is resolved to one of the two linear functions ( $a w$ or $b w$ ) when the position of $k v_{p}$ relative to $v_{m}$ and $v_{m+1}$ and the position of $k v_{p+1}$ relative to $v_{m+1}$ are determined (see Figure 3). Below, we partition the space of values into regions where the allocation is determined and payment is resolved to either $a w$ or $b w$. A region $V_{j, \text { positions }} k v_{p}$ between $v_{j}$ and $v_{j+1}$ determining allocation and payment for some of the agents. Payment for the rest of the agents is determined by 


\section{For the allocation problem with $m$ items and $n$ agents:}

1. let $K$ denote a finite set of values for the parameter $k$

2. for each threshold function $\tau_{k p}$ given by $k \in K$ and $p \in$ $\{1,2, \ldots, m-1\}$

- find the optimal rebate function $h_{k p}$ that satisfies Assumption 2

3. choose the mechanism $\left(\tau_{k p}, h_{k p}\right)$ with the highest welfare

Figure 2: Computational search for a welfare-maximizing mechanism.

$$
\begin{aligned}
& i \in\{1 \ldots p\} \\
& \quad f_{i}=1, t_{i}(v)=-\max \left(k v_{p+1}, v_{m+1}\right)+ \begin{cases}a v_{-i} & \text { if } k v_{p+1} \geq v_{m+1} \\
b v_{-i} & \text { otherwise }\end{cases} \\
& \quad \text { if } v_{i} \geq k v_{p}: f_{i}=1, t_{i}(v)=-\max \left(k v_{p}, v_{m+1}\right)+ \begin{cases}a v_{-i} & \text { if } k v_{p} \geq v_{m+1} \\
b v_{-i} & \text { otherwise }\end{cases} \\
& \text { otherwise: } f_{i}=0, t_{i}= \begin{cases}a v_{-i} & \text { if } k v_{p} \geq v_{m+1} \\
b v_{-i} & \text { otherwise }\end{cases} \\
& i \in\{(m+1) \ldots n\} \\
& f_{i}=0, t_{i}= \begin{cases}a v_{-i} & \text { if } k v_{p} \geq v_{m} \\
b v_{-i} & \text { otherwise }\end{cases}
\end{aligned}
$$

Figure 3: Mechanism satisfying Assumptions 1 and 2.

the second subscript which specifies whether $k v_{p+1}$ is above $(>)$ or below $(<)$ $v_{m+1}$.

$$
\begin{aligned}
\forall j \in\{p & \ldots m+1\} \\
V_{j,>}= & \left\{v \in V \mid v_{1} \geq \cdots \geq v_{p} \geq \cdots \geq v_{j} \geq k v_{p} \geq v_{j+1} \geq \cdots \geq v_{m} \geq \cdots \geq v_{n}\right. \\
& \left.\quad \operatorname{AND} k v_{p+1} \geq v_{m+1}\right\} \\
V_{j,<}= & \left\{v \in V \mid v_{1} \geq \cdots \geq v_{p} \geq \cdots \geq v_{j} \geq k v_{p} \geq v_{j+1} \geq \cdots \geq v_{m} \geq \cdots \geq v_{n}\right. \\
& \left.\quad \text { AND } k v_{p+1} \leq v_{m+1}\right\}
\end{aligned}
$$


The collection of regions above partitions the space $\left\{v \in V \mid v_{1} \geq v_{2} \geq\right.$ $\left.\ldots \geq v_{n} \geq 0\right\}$. We group constraints by region and restate the optimization problem (see Figure 4). Notice that on each region the constraints are of the form $d v \geq 0$ for some $d \in \mathbb{R}^{n}$, which means that they are satisfied at $\lambda v(\forall \lambda>0)$ as soon as they are satisfied at $v$. Hence we can assume without loss of generality that $v_{1}=1$ and focus on polytopes of vectors $\left(v_{2}, \ldots, v_{n}\right) \in \mathbb{R}^{n-1}$. A polytope $V_{j,>}$ (symmetrically $\left.V_{j,<}\right)$ is given by $(n+2)$ inequalities:

$$
1 \geq v_{2}, v_{2} \geq v_{3}, \cdots, v_{j} \geq \begin{gathered}
k v_{p}, k v_{p} \geq v_{j+1}, \cdots, v_{n-1} \geq v_{n}, v_{n} \geq 0 \\
k v_{p+1} \geq v_{m+1}
\end{gathered}
$$

The following fact tells us that it is enough to restrict attention to the extreme points of each of these polytopes.

Fact 1 . For any coefficients $a \in \mathbb{R}^{n}$ and $b \in \mathbb{R}$, a linear constraint $a v \geq b$ holds at all $v \in P$ of a polytope $P \subset \mathbb{R}^{n}$ if and only if it holds at the points $v \in$ ExtremePoints $(P)$, where ExtremePoints $(P)$ denotes the set of extreme points of polytope $P$.

Thus, making sure the constraints hold at the extreme points of $V_{j \text {, guar- }}$ antees that the constraints hold everywhere on the $V_{j}$. polytope. Now the linear program in Figure 4 can be solved by enforcing constraints only at the extreme points of each $V_{j}$, polytope.

Example As an example consider the allocation problem with $n=3, m=$ 2 and the threshold function specified by $k=.5$ and $p=1: \tau(w)=$ $\max \left(.5 w_{1}, w_{2}\right)$. The threshold function for agent 1 is $\max \left(.5 v_{2}, v_{3}\right)<v_{1}$ (see Figure 3 ). So agent 1 is always allocated an item. The threshold for agent 2 is $\max \left(.5 v_{1}, v_{3}\right)$. Agent 2 is allocated an item only when $v_{2} \geq .5 v_{1}$. Agent 3 is never allocated an item as the threshold for agent 3 is $\max \left(.5 v_{1}, v_{2}\right)>v_{3}$.

The rebate function is linear when in addition to allocation, the position of $.5 v_{1}$ and $.5 v_{2}$ relative to $v_{3}$ is determined. Taking $v_{1}=1$ we can represent this on a 2-dimensional graph (Figure 5 ). The space is divided into 5 regions, with each region having a linear rebate function and a fixed allocation. To make sure the constraints hold for all $\left\{v \in V \mid v_{1} \geq v_{2} \geq v_{3}\right\}$, we just need to enforce each region's constraints at its extreme points. For example, the extreme points of the right bottom region after adding $v_{1}=1$ as the first component are $(1, .5,0),(1, .5, .25),(1,1, .5),(1,1,0)$. 


$$
\begin{aligned}
\max _{a, b \in \mathbb{R}^{n-1}, r \in \mathbb{R}} r & \\
\forall j \in & \{p \ldots m+1\}, v \in V_{j,>} \\
& \sum_{i=1}^{j} v_{i}-\sum_{i=1}^{p} k v_{p+1}-\sum_{i=p+1}^{j} k v_{p}+\sum_{i=1}^{n} h\left(v_{-i} ; a, b\right) \geq r \sum_{i=1}^{m} v_{i} \\
& \sum_{i=1}^{n} h\left(v_{-i} ; a, b\right) \leq \sum_{i=1}^{p} k v_{p+1}+\sum_{i=p+1}^{j} k v_{p} \\
& h\left(v_{-i} ; a, b\right) \geq 0 \quad \forall i \\
\forall j \in & \{p \ldots m\}, v \in V_{j,<} \\
& \sum_{i=1}^{j} v_{i}-\sum_{i=1}^{p} v_{m+1}-\sum_{i=p+1}^{j} k v_{p}+\sum_{i=1}^{n} h\left(v_{-i} ; a, b\right) \geq r \sum_{i=1}^{m} v_{i} \\
& \sum_{i=1}^{n} h\left(v_{-i} ; a, b\right) \leq \sum_{i=1}^{p} v_{m+1}+\sum_{i=p+1}^{j} k v_{p} \\
& h\left(v_{-i} ; a, b\right) \geq 0 \quad \forall i
\end{aligned}
$$

Figure 4: Linear program with constraints grouped by regions $V_{j,>}$ and $V_{j,<}$.

We find mechanisms for different values of $n$ and $m$ using the computational procedure described in Figure 2. The class of threshold functions we consider is given by all pairs $(k, p)$ where $k$ takes values in $\{0, .025, .05, \ldots, .975\}$ and $p$ in $\{1,2, \ldots, m-1\}$. We used CPLEX 11.2.0 as a linear program solver.

For any fixed values of $n$ and $m$ we found that a mechanism with $p$ set to $m-1$ achieves the highest ratio. This setting of $p$ means that at most one item is destroyed. This result is consistent with the one obtained by Guo and Conitzer (2008) for randomized Groves mechanisms. They find that the best mechanism randomizes between destroying one item and not destroying any items.

The mechanisms we find provide the most improvement when the number of items is close to the number of agents. Our ratio gets closer to the ratio of the best Groves mechanism as the number of items gets smaller and approximately around $m=\frac{n}{2}$ the ratios and the mechanisms coincide. Figure 6 shows this trend for 10 agents and varying number of items. Also plotted 


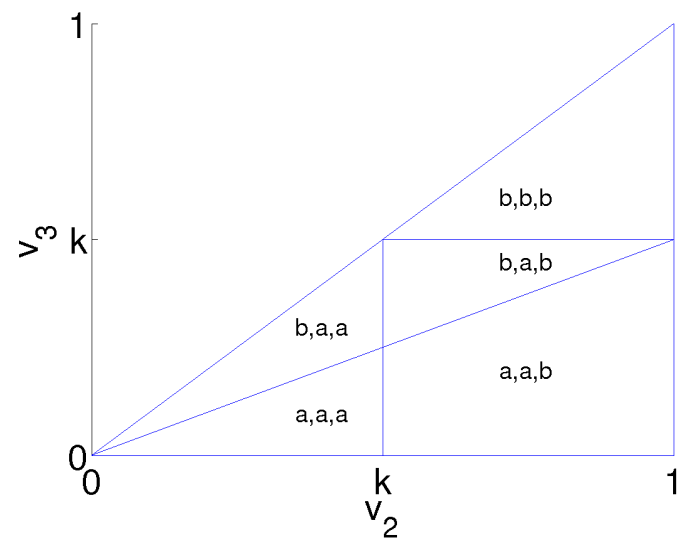

Figure 5: $\left(v_{1}=1\right)$ Regions where the number of allocated items remains constant and the rebate function is linear for 3 agents and 2 items. Each region is labeled with the coefficients used in the rebate function for each agent, e.g. (b,a,a) means that the rebate functions for agents 1,2 , and 3 are $b v_{-1}, a v_{-2}$, and $a v_{-3}$ respectively. One item is allocated to the left of the vertical line $v_{2}=k$ and two items to the right.

are the ratios achieved by the best VCG redistribution mechanism as well as the ratio achieved by the mechanism that first destroys a fixed number of items and then applies an optimal redistribution mechanism (see deterministic burning mechanism in Guo and Conitzer (2008)). All mechanisms coincide when the number of items is 4 or fewer.

\section{Analytical Results}

After analyzing mechanisms obtained numerically for various values of $n$ and $m$, we noticed a pattern and derived a simple mechanism parameterized by $n$ and $m$. We show that this mechanism, termed SimpleDestroy (SD), achieves the ratio of at least $1-\frac{\left(\begin{array}{c}n-m+1 \\ 2\end{array}\right)}{\left(\begin{array}{c}n \\ 2\end{array}\right)}$, which for any $m \geq .555 n$ is at least 0.8 asymptotically; moreover, when $m$ is close to $n$, the ratio approaches 1 .

\subsection{The SimpleDestroy mechanism}

The SimpleDestroy mechanism is defined in Figure 7. Note that it satisfies Assumptions 1 and 2 and is equivalently determined by the parameters $p=$ $m-1, k=\frac{n-m}{n}$ and the coefficients $a=(0, \ldots, 0), b=(0, \ldots, 0,-k, 1)$. 


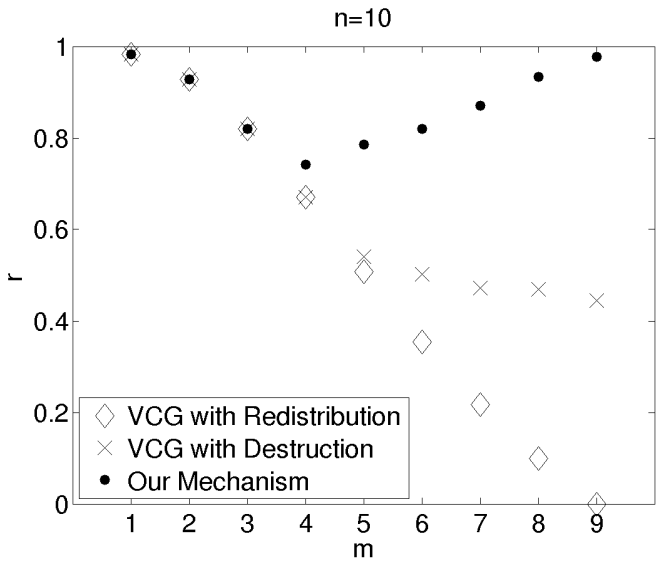

Figure 6: Performance of various mechanisms as a function of the number of items.

Intuitively, the mechanism is specified in two cases based on the amount of payment collected from the allocated agents before rebates. If the collected amount is small relative to the social welfare, then there are no rebates. This case occurs when the threshold is a fraction of the value of the last agent who is guaranteed to be allocated: $\tau(w)=k w_{m-1}$. On the other hand, when the threshold is above $k w_{m-1}$, the mechanism makes sure enough redistribution occurs if a significant portion of welfare is collected.

$$
\begin{aligned}
\tau(w)= & \max \left\{k w_{m-1} ; w_{m}\right\} \\
h(w)= & \begin{cases}0 & \text { if } \tau(w)=k w_{m-1} \\
w_{m}-k w_{m-1} & \text { if } \tau(w)=w_{m}\end{cases} \\
& \text { where } k=\frac{n-m}{n} .
\end{aligned}
$$

Figure 7: The SimpleDestroy Mechanism.

The next theorem proves that the SimpleDestroy mechanism satisfies the constraints and achieves good performance.

Theorem 2. The SimpleDestroy mechanism is individually rational, subsidyfree, and achieves the ratio of at least $1-\frac{\left(\begin{array}{c}n-m+1 \\ 2\end{array}\right)}{\left(\begin{array}{c}n \\ 2\end{array}\right)}$. 
Proof First note, that the rebate function is nonnegative, as its value is different from 0 only if $w_{m}>\frac{n-m}{n} w_{m-1}$, in which case $h(w)=w_{m}-\frac{n-m}{n} w_{m-1}>$ 0 . So, individual rationality always holds.

The threshold function $\tau(w)=\max \left\{\frac{n-m}{n} w_{m-1} ; w_{m}\right\}$ allows two allocations: agents $1 \ldots(m-1)$ are allocated when $v_{m}<\frac{n-m}{n} v_{m-1}$ and agents $1 \ldots m$ are allocated otherwise. We prove for each case separately.

(I) Assume $v_{m} \geq \frac{n-m}{n} v_{m-1}$, that is, $m$ items are allocated. This also defines the threshold and the rebates to agents $i=m+1, \ldots, n$ for which $\left(v_{-i}\right)_{m}=v_{m}$ and $\left(v_{-i}\right)_{m-1}=v_{m-1}$, and hence $\tau\left(v_{-i}\right)=v_{m}$ and $h\left(v_{-i}\right)=v_{m}-\frac{n-m}{n} v_{m-1}$. For agents $i=1, \ldots, m$, there are three possibilities as follows.

(Ia) If $v_{m+1} \geq \frac{n-m}{n} v_{m-1}$, then also $v_{m+1} \geq \frac{n-m}{n} v_{m}$, and thus for all $i=1, \ldots, m$ we have $\tau\left(v_{-i}\right)=v_{m+1}$. The rebates are given by $h\left(v_{-m}\right)=v_{m+1}-\frac{n-m}{n} v_{m-1}$ and $h\left(v_{-j}\right)=v_{m+1}-\frac{n-m}{n} v_{m}$ for $j=$ $1, \ldots, m-1$. Thereby, in this case we have

$$
\begin{aligned}
& \sum_{i=1}^{n} h\left(v_{-i}\right)-\sum_{i=1}^{m} \tau\left(v_{-i}\right) \\
& =\left[(m-1)\left(v_{m+1}-\frac{n-m}{n} v_{m}\right)+\left(v_{m+1}-\frac{n-m}{n} v_{m-1}\right)\right. \\
& \left.+(n-m)\left(v_{m}-\frac{n-m}{n} v_{m-1}\right)\right]-m v_{m+1} \\
& =\frac{(n-m)(n-m+1)}{n}\left(v_{m}-v_{m-1}\right) \leq 0,
\end{aligned}
$$

and so the SimpleDestroy mechanism is subsidy-free. The ratio 
is bounded as follows:

$$
\begin{aligned}
r^{\mathrm{SD}}(n, m) & =\frac{\sum_{i=1}^{m} v_{i}+\sum_{i=1}^{n} h\left(v_{-i}\right)-\sum_{i=1}^{m} \tau\left(v_{-i}\right)}{\sum_{i=1}^{m} v_{i}} \\
& =\frac{\sum_{i=1}^{m} v_{i}+\frac{(n-m)(n-m+1)}{n}\left(v_{m}-v_{m-1}\right)}{\sum_{i=1}^{m} v_{i}} \\
& =1+\frac{(n-m)(n-m+1)\left(v_{m}-v_{m-1}\right)}{n \sum_{i=1}^{m} v_{i}} \\
& \geq 1+\frac{(n-m)(n-m+1)\left(\frac{n-m}{n} v_{m-1}-v_{m-1}\right)}{n \sum_{i=1}^{m} v_{i}} \\
& =1-\frac{(n-m)(n-m+1) m v_{m-1}}{n^{2} \sum_{i=1}^{m} v_{i}} \geq 1-\frac{(n-m)(n-m+1) m}{n^{2}\left[(m-1)+\frac{n-m}{n}\right]} \\
& =1-\frac{(n-m)(n-m+1)}{n(n-1)}=1-\frac{\left(\begin{array}{c}
n-m+1 \\
2
\end{array}\right)}{\left(\begin{array}{c}
n \\
2
\end{array}\right)},
\end{aligned}
$$

where the inequalities follow from $v_{m} \geq \frac{n-m}{n} v_{m-1}$ and $v_{j} \geq$ $v_{m-1} \geq 0$ for $j=1, \ldots, m-1$.

(Ib) If $\frac{n-m}{n} v_{m-1}>v_{m+1} \geq \frac{n-m}{n} v_{m}$, then $\tau\left(v_{-m}\right)=\frac{n-m}{n} v_{m-1}$ and $h\left(v_{-m}\right)=0$. For agents $i=1, \ldots, m-1$ we have $\tau\left(v_{-i}\right)=v_{m+1}$ and $h\left(v_{-j}\right)=v_{m+1}-\frac{n-m}{n} v_{m}$, as before. In this case,

$$
\begin{aligned}
& \sum_{i=1}^{n} h\left(v_{-i}\right)-\sum_{i=1}^{m} \tau\left(v_{-i}\right) \\
& =\left[(m-1)\left(v_{m+1}-\frac{n-m}{n} v_{m}\right)+(n-m)\left(v_{m}-\frac{n-m}{n} v_{m-1}\right)\right] \\
& -\left[(m-1) v_{m+1}+\frac{n-m}{n} v_{m-1}\right] \\
& =\frac{(n-m)(n-m+1)}{n}\left(v_{m}-v_{m-1}\right) \leq 0,
\end{aligned}
$$

as required by the no-subsidy constraint. The bound on the ratio is achieved in the same way as in the previous case.

(Ic) Finally, if $\frac{n-m}{n} v_{m-1} \geq \frac{n-m}{n} v_{m} \geq v_{m+1}$ (with at least one inequality being strict), then all agents $i=1, \ldots, m$ get zero rebates, and the thresholds are $\tau\left(v_{-m}\right)=\frac{n-m}{n} v_{m-1}$ and $\tau\left(v_{-j}\right)=\frac{n-m}{n} v_{m}$ for 


$$
\begin{aligned}
j= & 1, \ldots, m-1 \text {. Now } \\
& \sum_{i=1}^{n} h\left(v_{-i}\right)-\sum_{i=1}^{m} \tau\left(v_{-i}\right) \\
& =(n-m)\left(v_{m}-\frac{n-m}{n} v_{m-1}\right)-\left[(m-1) \frac{n-m}{n} v_{m}+\frac{n-m}{n} v_{m-1}\right] \\
& =\frac{(n-m)(n-m+1)}{n}\left(v_{m}-v_{m-1}\right) \leq 0,
\end{aligned}
$$

so the no-subsidy holds. The ratio bound follows as in the previous case.

(II) Assume $v_{m}<\frac{n-m}{n} v_{m-1}$, that is, $m-1$ items are allocated. This also implies $v_{m+1}<\frac{n-m}{n} v_{m-1}$, and so for all agents $i=m, \ldots, n$ we have $\tau\left(v_{-i}\right)=\frac{n-m}{n} v_{m-1}$ and $h\left(v_{-i}\right)=0$. For agents $i=1, \ldots, m-1$, there are two possibilities as follows.

(IIa) If $v_{m+1} \geq \frac{n-m}{n} v_{m}$, then $\tau\left(v_{-i}\right)=v_{m+1}$ and $h\left(v_{-i}\right)=v_{m+1}-\frac{n-m}{n} v_{m}$ for all $i=1, \ldots, m-1$. Thus, in this case we have

$$
\begin{aligned}
& \sum_{i=1}^{n} h\left(v_{-i}\right)-\sum_{i=1}^{m-1} \tau\left(v_{-i}\right) \\
& =(m-1)\left(v_{m+1}-\frac{n-m}{n} v_{m}\right)-(m-1) v_{m+1}=-\frac{(m-1)(n-m)}{n} v_{m} \leq 0
\end{aligned}
$$

and so the SimpleDestroy mechanism is subsidy-free. The ratio is bounded as follows:

$$
\begin{aligned}
r^{\mathrm{SD}}(n, m) & =\frac{\sum_{i=1}^{m-1} v_{i}+\sum_{i=1}^{n} h\left(v_{-i}\right)-\sum_{i=1}^{m-1} \tau\left(v_{-i}\right)}{\sum_{i=1}^{m} v_{i}} \\
& =\frac{\sum_{i=1}^{m-1} v_{i}-\frac{(m-1)(n-m)}{n} v_{m}}{\sum_{i=1}^{m} v_{i}}=\frac{\sum_{i=1}^{m} v_{i}-\frac{(m-1)(n-m)}{n} v_{m}-v_{m}}{\sum_{i=1}^{m} v_{i}} \\
& =1-\frac{m(n-m+1) v_{m}}{n \sum_{i=1}^{m} v_{i}}>1-\frac{m(n-m+1)}{n\left((m-1) \frac{n}{n-m}+1\right)} \\
& =1-\frac{(n-m)(n-m+1)}{n(n-1)}=1-\frac{\left(\begin{array}{c}
n-m+1 \\
2
\end{array}\right)}{\left(\begin{array}{c}
n \\
2
\end{array}\right)},
\end{aligned}
$$

where the inequality is implied by $v_{i} \geq v_{m-1}>\frac{n}{n-m} v_{m} \geq 0$ for $i=1, \ldots, m-1$. 
(IIb) If $v_{m+1}<\frac{n-m}{n} v_{m}$, then $\tau\left(v_{-i}\right)=\frac{n-m}{n} v_{m}$ and $h\left(v_{-i}\right)=0$ for all $i=1, \ldots, m-1$. In this case,

$$
\begin{aligned}
& \sum_{i=1}^{n} h\left(v_{-i}\right)-\sum_{i=1}^{m-1} \tau\left(v_{-i}\right) \\
& =0-(m-1) \frac{n-m}{n} v_{m}=-\frac{(m-1)(n-m)}{n} v_{m} \leq 0,
\end{aligned}
$$

so the no-subsidy holds, and the ratio bound follows as before.

The proof is now complete.

Recall that the ratio of the optimal Groves mechanism is close to 1 when the number of items is small. However, it is not difficult to check that it decreases as the number of items increases, and reaches 0 once the number of items is as high as it can be: one fewer than the number of agents. Indeed, this ratio, derived by Moulin (2009) and Guo and Conitzer (2009), is

$$
r^{\mathrm{VCG}}(n, m)=1-\frac{\left(\begin{array}{c}
n-1 \\
m
\end{array}\right)}{\sum_{j=m}^{n-1}\left(\begin{array}{c}
n-1 \\
j
\end{array}\right)}
$$

On the other hand, as shown above, the ratio of SimpleDestroy increases with the number of items. For each $n$, there exists a unique integer $m^{*}<n$ such that SimpleDestroy overtakes the optimal Groves mechanism, whenever there are at least $m^{*}$ items to allocate. While there is no simple closed-form expression of $m^{*}$ as a function of $n$, it is simple enough to compute it numerically for specific values of $n$, using the expressions of $r^{\mathrm{VCG}}$ and $r^{\mathrm{SD}}$. We observe that $m^{*}$ seems to always fall in the neighborhood of $1 / 2$ (see Figure 8). SimpleDestroy performs best when the number of items is the largest, i.e. when the optimal Groves mechanism performs the worst. This motivates the definition of the hybrid mechanism, which uses the optimal Groves mechanism when the number of items is less than $m^{*}$ and SimpleDestroy otherwise.

We now study the limit case when both $m$ and $n$ are large. The purpose of this asymptotic analysis is to better understand how SimpleDestroy and optimal Groves compare, and in which circumstances to employ one or the other in the hybrid mechanism. Consider two increasing sequences $\left(n_{q}\right)$ and $\left(m_{q}\right)$ of positive integers such that $m_{q}<n_{q}$ for all $q$, the number $n_{q} \rightarrow \infty$ when $q \rightarrow \infty$, and the sequence $\left(\frac{m_{q}}{n_{q}}\right)$ converges to some $\alpha \in(0,1)$ (thus to be interpreted as the maximal percentage of the population that could receive an item). 


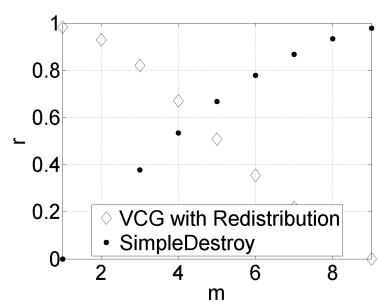

(a) $n=10$

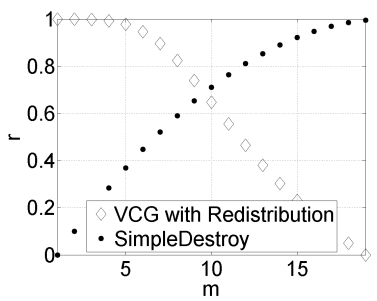

(b) $n=20$

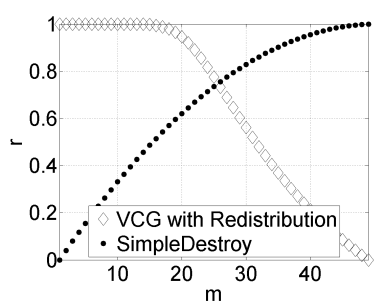

(c) $n=50$

Figure 8: Performance of optimal Groves with Redistribution and SimpleDestroy mechanisms.

\section{Proposition 1.}

1.

$$
r^{V C G}(\alpha):=\lim _{q \rightarrow \infty} r^{V C G}\left(n_{q}, m_{q}\right)= \begin{cases}1 & \alpha \leq 1 / 2 \\ \frac{1-\alpha}{\alpha} & \alpha>1 / 2\end{cases}
$$

(see Moulin (2009), Theorem 3).

2. $r^{S D}(\alpha):=\lim _{q \rightarrow \infty} r^{S D}\left(n_{q}, m_{q}\right)=2 \alpha-\alpha^{2}$.

3. The asymptotically best hybrid mechanism is obtained by choosing $m^{*} \sim$ $0.555 n$. It guarantees a ratio of at least 0.8 for any $\alpha$.

Proof Note that

$$
r^{\mathrm{SD}}(n, m)=1-\frac{(n-m)(n-m+1)}{n(n-1)}=\frac{2(m-1)}{n-1}-\frac{m(m-1)}{n(n-1)}
$$

Hence,

$$
\lim _{q \rightarrow \infty} r^{\mathrm{SD}}\left(n_{q}, m_{q}\right)=\lim _{q \rightarrow \infty}\left(\frac{2\left(m_{q}-1\right)}{n_{q}-1}-\frac{m_{q}\left(m_{q}-1\right)}{n_{q}\left(n_{q}-1\right)}\right)=2 \alpha-\alpha^{2}
$$

The asymptotically best hybrid mechanism and its associated guaranteed ratio are then obtained by solving the equation $\frac{1-m^{*}}{m^{*}}=2 m^{*}-\left(m^{*}\right)^{2}$.

Notice that $r^{\mathrm{SD}}(\cdot)$ is non-negative and monotonically increasing for $\alpha$ between 0 and 1 ; moreover, it is concave (quadratic in $\alpha$ ), and so the ratio becomes quickly higher for relatively low $\alpha$ 's. The function $r^{\mathrm{VCG}}(\cdot)$, on the other hand, is monotonically decreasing and convex when $\alpha>1 / 2$, implying that the ratio becomes quickly lower for $\alpha$ 's larger than $1 / 2$. For instance, if there are enough items to serve $75 \%$ of the population, then SimpleDestroy asymptotically guarantees the ratio of $\frac{15}{16}$, while the optimal Groves mechanism asymptotically guarantees the ratio of only $1 / 3$. 


\section{Discussion}

Allocative efficiency of Groves mechanisms conflicts with social welfare in the fundamental allocation model we consider. This means a lot of money may need to be burnt to maintain strategy-proofness, especially when the proportion of allocated agents is high. In particular, the social welfare is lost completely when there are enough items for all but one agent, and all agents have the same value for consuming an item. In this case, the Groves payment equals the value, and no redistribution is possible (Moulin, 2009; Guo and Conitzer, 2009).

It turns out that a small departure from efficiency lets us recover most of the loss. Specifically, this paper presents the SimpleDestroy mechanism that sometimes does not allocate to the last agent who would be allocated under an efficient mechanism, and guarantees a high level of social welfare when the number of items is at least half the number of agents. In contrast to efficient mechanisms, the welfare guaranteed by this mechanism increases with the percentage of allocated agents, and rapidly goes to 1 as the number of items approaches the number of agents. Furthermore, it follows that a hybrid mechanism applying the optimal Groves mechanism for $m<.555 n$ and SimpleDestroy otherwise, guarantees a high level of social welfare (asymptotic ratio of at least 0.8 ) for all allocation instances.

Our results are guided by an algorithmic procedure that exploits linearity inherent in the model. Specifically, we restrict attention to a class of mechanisms where optimization can be performed via linear programming, and numerically find mechanisms which are optimal within this restricted class. ${ }^{13}$ In many cases, these mechanisms guarantee social welfare which is close to the total social welfare, and thus no further significant improvement is possible. However, they are not generally optimal. For instance, for the problem with 3 agents and 2 items we were able to find the following provably optimal

\footnotetext{
${ }^{13} \mathrm{~A}$ similar methodology was used in (Guo and Conitzer, 2010a) to derive optimal payments when free items are efficiently allocated. More generally, algorithmic approaches to mechanism design problems are considered in (Conitzer and Sandholm, 2002; Guo and Conitzer, 2010a).
} 
mechanism: ${ }^{14}$

$$
\begin{aligned}
& \tau(w)=\max \left(\frac{1}{4}\left(w^{1}+w^{2}\right), w^{2}\right) \\
& h(w)= \begin{cases}\frac{2}{32} w^{2} & \text { if } \frac{1}{9} w^{1} \geq w^{2} \\
-\frac{10}{32} w^{1}+\frac{11}{32} w^{2} & \text { if } \frac{1}{3} w^{1} \geq w^{2} \geq \frac{1}{9} w^{1} \\
-\frac{4}{32} w^{1}+\frac{20}{32} w^{2} & \text { otherwise }\end{cases}
\end{aligned}
$$

This mechanism guarantees the ratio of .75 , which is higher than what can be obtained for 3 agents and 2 items by the mechanisms within the restricted class we considered. The challenge is then to find a general mechanism that is provably optimal for any number of items and agents. A few simpler questions will need to be answered along the way: is the natural property that if an agent with value $v_{i}$ is allocated then all agents with values above $v_{i}$ are also allocated, consistent with an optimal mechanism? Is destroying more than one item ever beneficial? Another interesting open question is whether a broader class of mechanisms would provide a significant asymptotic improvement for general problem instances. A different avenue for future work is investigating destruction in other models. For instance, an efficient mechanism for allocating heterogeneous items (Gujar and Narahari, 2011) has recently been proven optimal by Guo (2012). Designing inefficient mechanisms with better welfare guarantees in this heterogeneous model is an open question. Another class of problems where destruction mechanisms may be beneficial is online allocation mechanisms (Parkes, 2007).

Bailey, M.J., 1997. The demand revealing process: To distribute the surplus. Public Choice 91, 107-26.

Cavallo, R., 2006. Optimal decision-making with minimal waste: Strategyproof redistribution of vcg payments, in: AAMAS'06, Hakodate, Japan. pp. 882-889.

de Clippel, G., Naroditskiy, V., Greenwald, A., 2009. Destroy to save, in: EC'09, pp. 207-214.

Conitzer, V., Sandholm, T., 2002. Complexity of mechanism design, in: UAI, pp. $103-110$.

\footnotetext{
${ }^{14}$ The optimality proof of this mechanism can be found in (Naroditskiy, 2009).
} 
Dufton, L., Naroditskiy, V., Polukarov, M., Jennings, N.R., 2012. Optimizing payments in dominant-strategy mechanisms for multi-parameter domains, in: AAAI, pp. 1347-1354.

Faltings, B., 2005. A Budget-balanced, Incentive-compatible Scheme for Social Choice, in: Faratin, P., Rodriguez-Aguilar, J.A. (Eds.), AgentMediated Electronic Commerce VI. Springer. volume 3435, pp. 30-43.

Green, J.R., Laffont, J.J., 1979. Incentives in public decision-making. North Holland, New York.

Gujar, S., Narahari, Y., 2011. Redistribution mechanisms for assignment of heterogeneous objects. Journal of Artificial Intelligence Research 41, $131-154$.

Guo, M., 2012. Worst-case optimal redistribution of VCG payments in heterogeneous-item auctions with unit demand, in: AAMAS, pp. 745-752.

Guo, M., Conitzer, V., 2008. Better redistribution with inefficient allocation in multi-unit auctions with unit demand, in: EC'08, pp. 210-219.

Guo, M., Conitzer, V., 2009. Worst-case optimal redistribution of vcg payments in multi-unit auctions. Games and Economic Behavior 67, $69-$ 98.

Guo, M., Conitzer, V., 2010a. Computationally feasible automated mechanism design: General approach and case studies, in: AAAI, pp. 1676-1679.

Guo, M., Conitzer, V., 2010b. Optimal-in-expectation redistribution mechanisms. Artif. Intell. 174, 363-381.

Guo, M., Naroditskiy, V., Conitzer, V., Greenwald, A., Jennings, N.R., 2011. Budget-balanced and nearly efficient randomized mechanisms: Public goods and beyond, in: WINE, pp. 158-169.

Moulin, H., 2009. Almost budget-balanced vcg mechanisms to assign multiple objects. Journal of Economic Theory 144, 96-119.

Naroditskiy, V., 2009. Select Problems at the Intersection of Computer Science and Economics. Ph.D. thesis. Brown University. 
Naroditskiy, V., Polukarov, M., Jennings, N.R., 2012. Optimization of payments in dominant strategy mechanisms for single-parameter domains. ACM Transactions on Economics and Computation (to appear) .

Nisan, N., Roughgarden, T., Tardos, E., Vazirani, V.V., 2007. Algorithmic Game Theory. Cambridge University Press, New York, NY, USA.

Parkes, D.C., 2007. Online mechanisms, in: Nisan, N., Roughgarden, T., Tardos, E., Vazirani, V. (Eds.), Algorithmic Game Theory, pp. 411-439.

Porter, R., Shoham, Y., Tennenholtz, M., 2004. Fair imposition. Journal of Economic Theory 118, $209-228$. 